\title{
An Orally Bioavailable (Mice) Prodrug of Glutathione
}

\author{
Daune L. Crankshaw ${ }^{1}$, Jacquie E. Briggs ${ }^{1}$, Robert Vince ${ }^{2}$ and Herbert T. Nagasawa ${ }^{1,3,}$, \\ 1 Center for Drug Design, University of Minnesota. Minneapolis, MN 55455 \\ 2. Director, Center for Drug Design \\ 3 Department of Veterans Affairs Medical Center, One Veterans Drive, Minneapolis, MN 55417 \\ * Correspondence: nagas001@umn.edu. Tel. US 949-854-6125
}

\begin{abstract}
Cysteine-glutathione mixed disulfide (CySSG), a prodrug of glutathione (GSH) --the "Master Antioxidant", was found to be orally bioavailable in mice, and protected against a toxic dose of acetaminophen. If oral bioavailability can also be demonstrated in humans, this suggests a wide range of applicability for CySSG.
\end{abstract}

Keywords: CySSG; prodrug; glutathione; orally; bioavailable

\section{Introduction}

In an earlier publication [1], we reported that the mixed disulfide of L-cysteine and GSH, viz., CySSG (Sis-Gee), protected mice against acetaminophen-induced hepatotoxicity when administered intraperitoneally. We now report that CySSG, a prodrug of GSH which is naturally found in human blood [2] also protected mice against acetaminophen overdose when administered by oral gavage. On reduction of its disulfide bond in vivo [3] CySSG releases not only GSH but also an equivalent of L-cysteine, the rate-limiting amino acid required for the de novo biosynthesis of GSH.

\section{Materials and Methods}

Fasted (12 hrs) male, Swiss-Webster mice (numbers indicated in Figure 1) were administered $2.45 \mathrm{mmol} / \mathrm{kg}$ of ACP intraperitoneally. This was followed $30 \mathrm{~min}$. later with $2.50 \mathrm{mmol} / \mathrm{kg}$ of CySSG orally by gavage. The mice were sacrificed $24 \mathrm{hrs}$ post-ACP, and blood was withdrawn for determination of plasma ALT levels. The data were plotted in a manner that allowed ready comparison between data [4]. The use of mice for this experiment was approved by the University of Minnesota Animal Care and Use Committee (ACUC).

\section{Results}

Figure 1 describes the data which show the efficacy of orally administered CySSG in protecting mice against a toxic dose of acetaminophen. Note in the figure that the ordinates are in logarithmic scale to condense the chart $(\log 2=100 ; \log 4=10,000)$, and reflect the serum ALT levels of the mice at 24 hrs post acetaminophen, a quantitative indication of hepatic damage. The numbers of survivors are indicated over the data.

The hatched areas of the vertical bars reflect the $99 \%$ confidence interval, while the unhatched (clear) extension of the bars reflect the $95 \%$ confidence interval of the log-transformed ALT values pursuant to [4]. Thus, a horizontal intersect of the hatched areas of any two data bars indicates that the data are identical at the $99 \%$ level of confidence; whereas a horizontal intersect encompassing the unhatched areas indicates that the data are identical at the $95 \%$ confidence interval.

It can be seen that the ACP Control mice had some toxic deaths (2 of 13), as well as severe elevations in blood ALT levels indicated by the large log values, whereas mice given identical ACP doses plus oral CySSG survived with essentially normalized blood 
ALT levels. The " $n$ " for CySSG in this protocol was 27 (9 x 3), representing three independent experiments.

Figure 1

\section{Alanine Transaminase (ALT) Levels of Mice Treated Orally with CySSG}

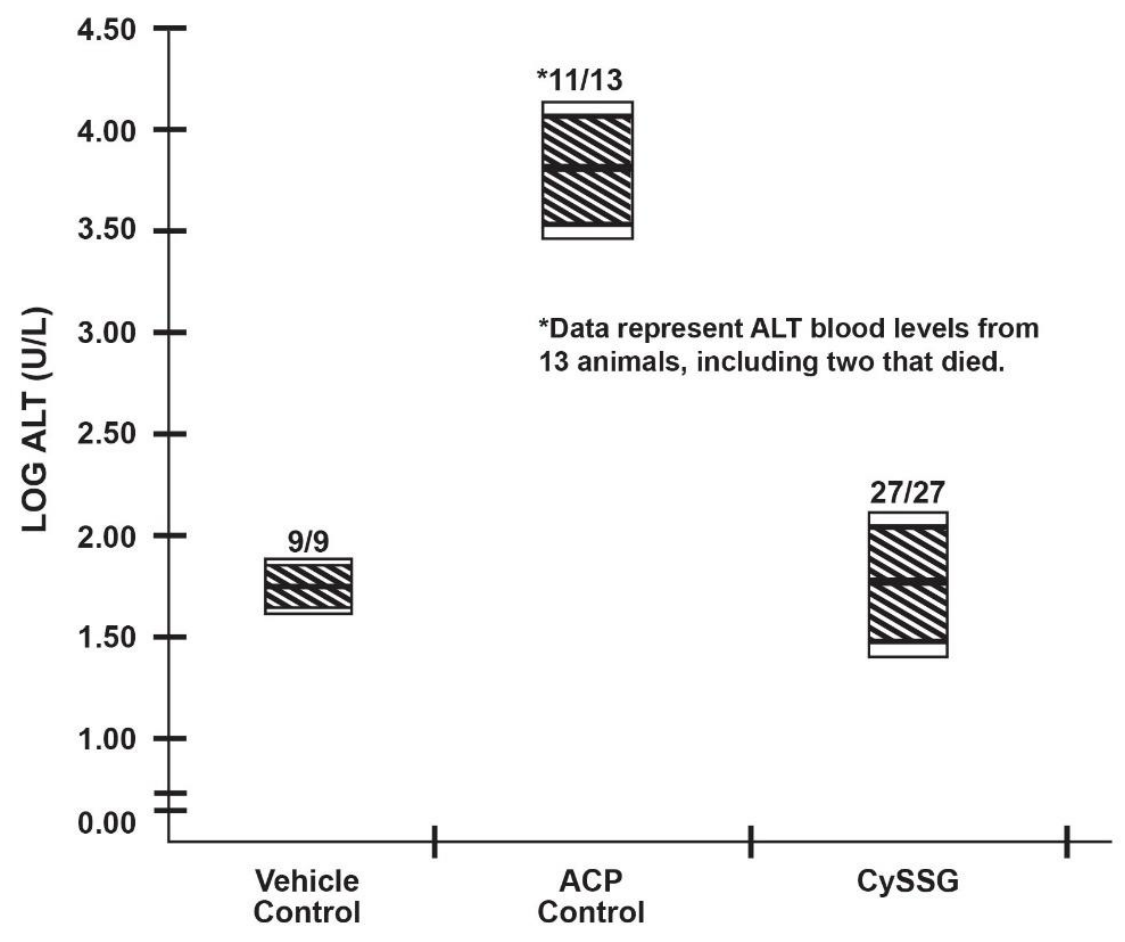

\section{Discussion}

Oral administration of CySSG to mice treated with a toxic dose of acetaminophen fully protected the animals, as indicated by their serum ALT levels which were not different from the vehicle controls (Fig.1). Thus, CySSG has now been shown to be orally bioavailable for the delivery of GSH in this mouse model, and while mice and rat data are generally transferable to humans, it is incumbent that human studies be conducted as soon as possible by clinician investigators. GSH taken orally by humans is degraded by the enzyme, gamma-glutamyl transpeptidase, and is known not to be bioavailable [5], whereas the mechanism that releases GSH from CySSG intracellularly is via a thiol-disulfide exchange reaction [3].

It is known that the first enzyme in the two-step de novo biosynthetic pathway for GSH is greatly compromised in people older than 60 years [6]; hence, CySSG, if found to release and provide preformed GSH in humans, should be of great value for our older generation. Moreover, GSH delivered via orally bioavailable CySSG may be more effective than nebulized GSH [7] for Covid-infected patients. Further interest would be to compare oral CySSG with intravenous GSH [7] in relieving the severity of Covid-19.

Patents: The Dept. of Veterans Affairs (DVA), Washington DC, has assigned the patent rights for CySSG to a commercial entity for further development.

Funding: This research was funded by the Dept of Veterans Affairs, Minneapolis, MN, and the Center for Drug Design, University of Minnesota.

Conflicts of Interest: HTN serves as science consultant for the Deptment of Veterans Affairs and the company mentioned above.

Acknowledgments: We thank Sccot Momii, MBA, for converting this manuscript into the reqired template format and other services. 


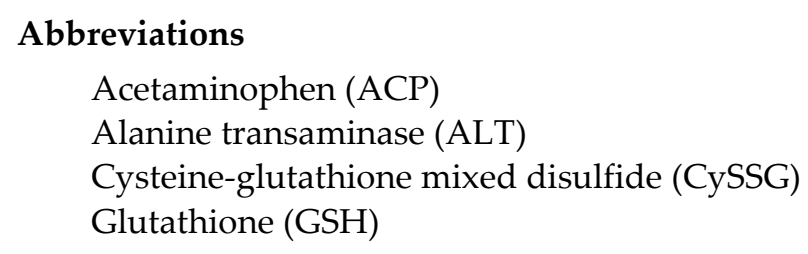

\section{References}

1. Berkeley, L.I.; Cohen, J.F.; Crankshaw, D.L.; Shirota, F.N.; Nagasawa, H.T. Hepatoprotection by L-cysteine-glutathione mixed disulfide. A sulfhydryl-modified prodrug of glutathione. J. Biochem. Mol. Toxicol. 2003, 17, 95-97.

2. Kleinman, W.A.; Richie, J.P. Jr. Status of glutathione and other thiols in human plasma. Biochem. Pharmacol. 2000, 6, 19-29.

3. Eriksson, S.A.; Mannervik, B. The reduction of the L-cysteine-glutathione mixed disulfide in rat liver. Involvement of an enzyme catalyzing thiol-disulfide interchange. FEBS LET 7, No. 2, 1970, 7, 26-28.

4. Nagasawa, H.T.; Shoeman, D.W.; Cohen, J.F.; Rathbun, W.B. Protection against acetaminophen-induced hepatotoxicity by Lcyssme and its N-acetyl and ethyl ester derivatives. J. Biochem. Mol. Toxicol. 1996, 11, 289-295.

5. Witschi, A.; Reddy, S.; Stofer, B.; Lauterburg, R.H. The systemic availability of oral glutathione. Eur. J. Pharmacol. 1992, 43, 667-669.

6.Van Lieshout, E.M.M.; Peters, W.H.M. Age and gender dependent levels of glutathione and glutathione-S-transferase in human lymphocytes. Carcinogenesis 1998, 19, 1873-1875.

7. Silvagno, F.; Vermone, A.; Pescamona, G.P. The role of glutathione in protecting against the severe inflammatory response triggered by COVD-19. Antioxidants (Basel) 2020, 9, 24. 